\title{
High Resolution Scanning Transmission Electron Microscopy of Normal and Inverse Spinel Regions in Epitaxially Grown $\mathrm{CoFe}_{2} \mathrm{O}_{4}$
}

Amanda Hanks Trout ${ }^{1,2}$, Igor V. Pinchuk ${ }^{3}$, Walid Amamou ${ }^{3}$, Robert E. A. Williams ${ }^{1}$, Roland K. Kawakami $^{3}$ and David W. McComb ${ }^{1,2}$

${ }^{1 .}$ Center for Electron Microscopy and Analysis, The Ohio State University, Columbus OH.

2. Department of Materials Science and Engineering, The Ohio State University, Columbus OH.

3. Department of Physics, The Ohio State University, Columbus OH.

Oxide spinels are an interesting class of materials due to their diverse chemical, magnetic, and electric properties. ${ }^{1,2}$ The variation in cation species and coordination environment has a substantial impact on the material properties and has been investigated extensively using various techniques, including electron microscopy. ${ }^{3,4}$ Here we discuss the investigation of $\mathrm{CoFe}_{2} \mathrm{O}_{4}$ (CFO), nominally an inverse spinel ferromagnetic insulator that has been shown to be promising for magnetic proximity effect (MPE) and spin manipulation, grown via molecular beam epitaxy (MBE) through high-resolution scanning transmission electron microscopy (STEM). ${ }^{5}$

The general formula of spinel materials is $\mathrm{AB}_{2} \mathrm{O}_{4}$ with a unit cell of space group $\mathrm{Fd}-3 \mathrm{~m}$. There are two forms of the spinel structure: normal and inverse. In the normal spinel, O ions make up an FCC lattice with $1 / 8$ of the tetrahedral holes occupied by divalent ions and $1 / 2$ of the octahedral holes occupied by trivalent ions. Inverse spinels have half of the trivalent atoms on tetrahedral positions and the divalent atoms on octahedral positions and have the general formula of $\mathrm{B}(\mathrm{AB}) \mathrm{O}_{4}$. Few inverse spinel materials meet this criterion, however, and rather have a fraction of the tetrahedral sites occupied by a trivalent atom. This fraction is known as the degree of inversion and is denoted by $\lambda$. A fully inverse spinel would have $\lambda=1$ and normal spinel $\lambda=0 .{ }^{4,6}$ Cobalt ferrite is not a fully inverse spinel, and has a $\lambda$ value of $\sim 0.75$ $0.8 .^{1}$

High angle annular dark field (HAADF) image in Figure 1a shows the quality of the MBE grown thin film. The interface between the substrate and thin film is atomically sharp with an epitaxial relationship of [100] // [100] and [010] // [010]. Additionally, there is no evidence of defects through the CFO layer. While some areas in the cross sectional HAADF images exhibit contrast consistent with the expected inverse spinel structure, there are also regions where the contrast is quite different. (Figure 1) This variation in the contrast is consistent with a mixture of normal and inverse spinel regions. (Figure 1b)

$\mu \mathrm{STEM}$, a S/TEM simulation program based on inelastic scattering, ${ }^{7}$ was used to simulate the HAADF images of fully normal and fully inverse structures of CFO and are shown in Figure 2. The simulated HAADF images confirm that there is a difference between normal and inverse spinel structures. These simulations suggest that channeling effects are significant enough to differentiate between these regions. In this contribution, we will further discuss using STEM techniques to investigate normal and inverse spinel regions of MBE grown CFO. 
References:

[1] Ferreira, T. A. S. et al, Solid State Sciences 5, (2003), p. 383.

[2] Tatarchuk, T. R. et al, Journal of Alloys and Compounds 694 (2017), p. 777.

[3] Docherty, F. T. et al, Ultramicroscopy 86 (2001), p. 273.

[4] Tileli, V. et al, Nanoscale 7 (2015), p. 218.

[5] Amamou, W. et al, Physical Review Materials 2, (2018).

[6] Gobbi, G. C. et al, Chemistry Letters 14 (1985), p. 771.

[7] Allen, L. J. et al, Ultramicroscopy 151 (2015), p. 11.

a

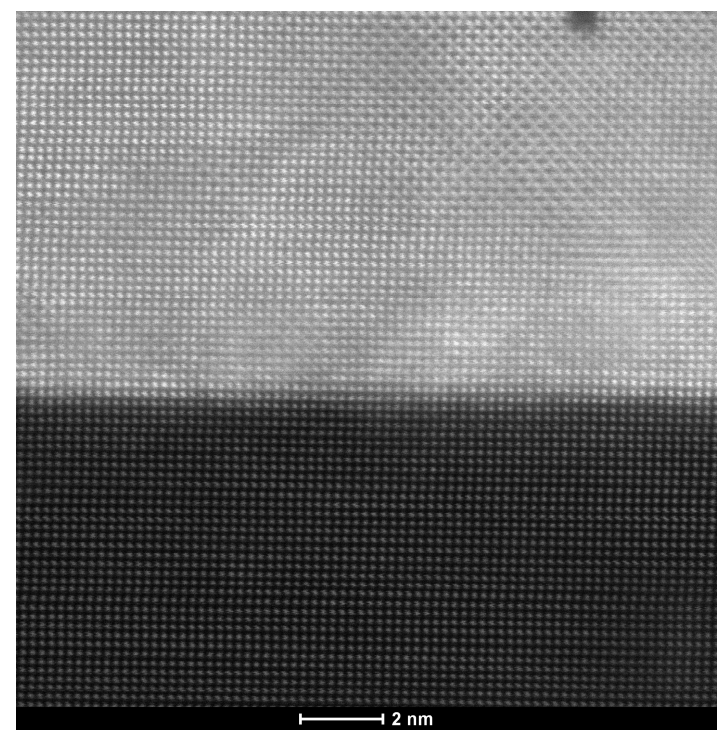

b

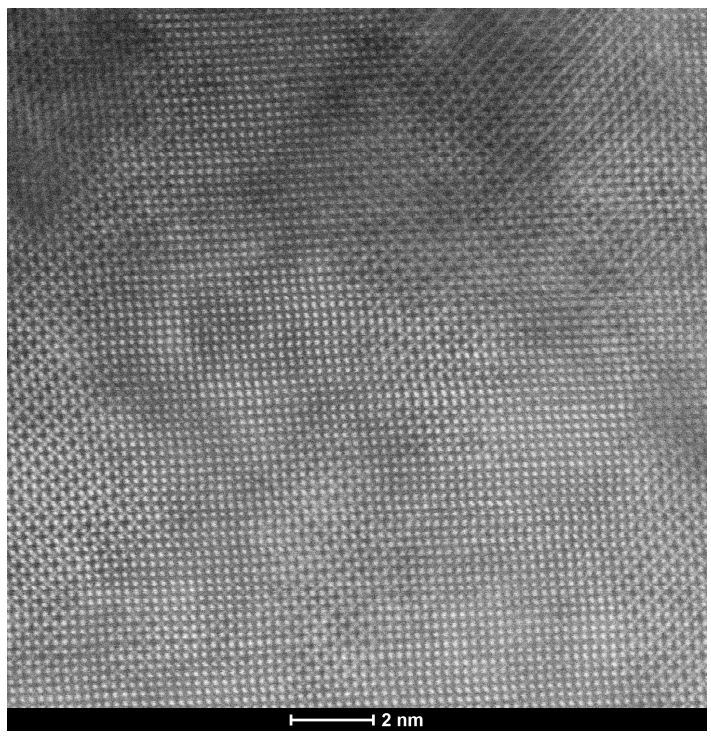

Figure 1. Cross-sectional HADDF images of (a) the $\mathrm{CFO} / \mathrm{MgO}$ interface and (b) anisotropic growth of normal and inverse spinel regions in the CFO layer.
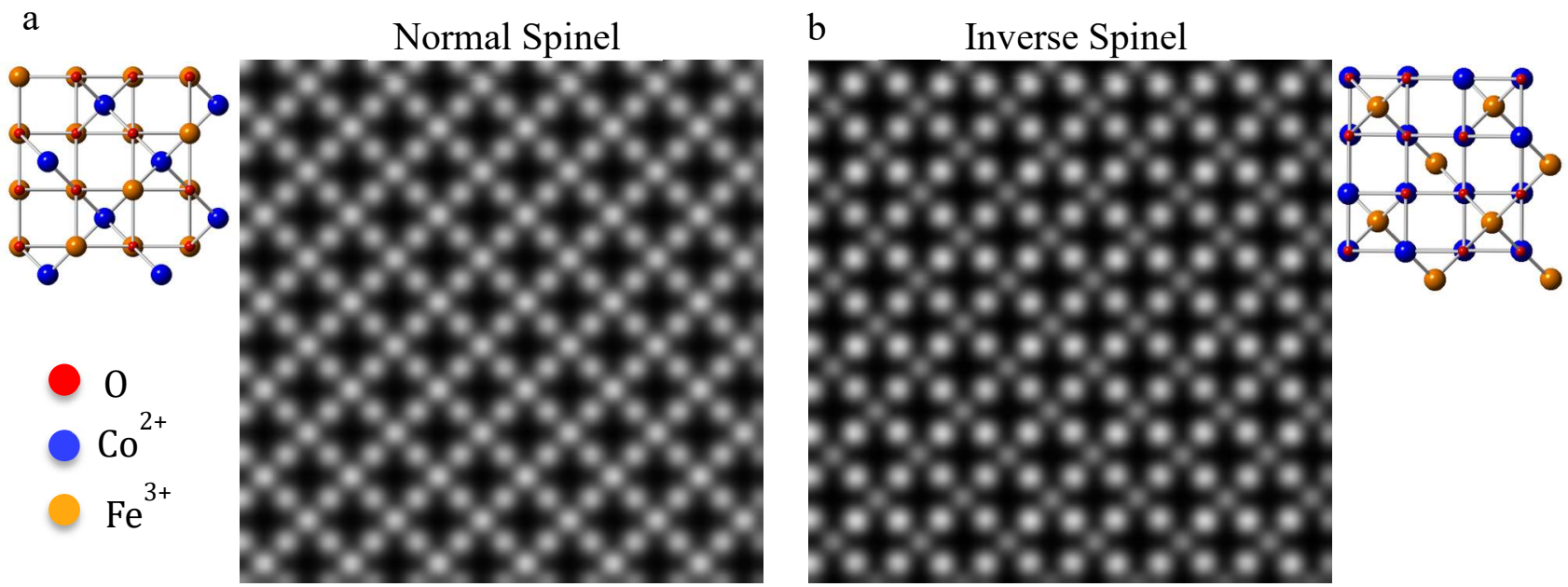

Figure 2. Simulated HAADF images of (a) normal and (b) fully inverse spinel cobalt ferrite. 